\title{
Composition anatomique de canards mulards mâles de 4 génotypes : influence de l'âge et du gavage
}

\author{
JM Brun 1, G Guy 2, B Poujardieu 1, \\ D Rousselot-Pailley ${ }^{2}$, R Rouvier 1
}

1 INRA, station d'amélioration génétique des animaux, BP 27, 31326 Castanet-Tolosan cedex;

2 INRA, station expérimentale des palmipèdes à foie gras, Artiguères, 40280 Benquet, France

(Reçu le 7 mars 1994 ; accepté le 26 septembre 1994)

\begin{abstract}
Résumé - La composition anatomique de 216 canards mulards mâles contemporains issus de 4 types génétiques de mères a été étudiée sur des animaux non gavés abattus aux âges de 12 et de 14 sem et sur des animaux gavés âgés de 14 sem. Les types génétiques de mères proviennent d'un croisement factoriel entre une souche de type Pékin Européen (P) et une souche de Tsaiya Brune (T), dont le poids adulte est de 3 et $1,4 \mathrm{~kg}$ respectivement. Aux 3 stades, l'influence du type génétique est significative pour presque toutes les caractéristiques étudiées. Aux âges de 12 et $14 \mathrm{sem}$, le classement des types génétiques sur le poids de la plupart des éléments de la carcasse est le suivant $: P>P$ $\times T=T \times P>T$. Ce classement vaut également pour le pourcentage par rapport au poids vif de différents dépôts adipeux (gras sous-cutané de la cuisse et des pectoraux, gras abdominal) et des muscles grand-pectoraux. II s'inverse pour le pourcentage de muscle de l'ensemble cuisse + pilon. La plupart des éléments de la carcasse croissent entre les âges de 12 et 14 sem sauf le foie, les pattes, le muscle et l'os de l'ensemble cuisse + pilon. Les mulards de Tsaiya ont la croissance relative la plus élevée du poids vif à l'abattage, du poids de la carcasse, du poids des ailes, du poids des filets et de ses composantes (peau + gras sous-cutané, muscles grand-pectoraux). Par rapport au poids vif, la proportion de divers dépôts adipeux augmente entre 12 et 14 sem ainsi que celle des muscles pectoraux alors que celle des muscles de la cuisse diminue. Dans les 4 génotypes, le gavage augmente significativement le poids du foie qui, en moyenne sur les 4 types génétiques, passe de 55 à $583 \mathrm{~g}$, le poids du gras abdominal (de 43 à $150 \mathrm{~g}$ ), le poids de l'ensemble "peau + gras" de la cuisse (de 73 à $177 \mathrm{~g}$ ) et de celui des magrets (de 126 à $294 \mathrm{~g}$ ). Ces derniers représentent l'ensemble «muscles grand-pectoraux + peau + gras sous-cutané». Le gavage augmente aussi le poids de muscle de la cuisse (de 149 à $171 \mathrm{~g}$ ) mais pas celui des pectoraux.
\end{abstract}

canard mulard / croissance / composition anatomique / gavage / croisement

Summary - Anatomical composition of male mule ducklings of 4 genotypes : age and cramming effects. The anatomical composition of 216 contemporaneous male mule ducklings representing 4 breed types of dams was assessed at 3 stages: 12 and 14 weeks of age for normally-fed animals 
and 14 weeks of age for force-fed animals. The 4 types of dams came from a factorial crossbreeding design between a strain of the "European Pekin" type $(P)$ and another of the "brown Tsaiya" type (T), their adult body weight being 3 and $1.4 \mathrm{~kg}$, respectively. Almost all the traits studied were significantly affected by genotype. For most of the carcass component weights, the ranking of the breed types was: $P>P \times T=T \times P>T$. This ranking was also true for live weight percentage of various fat deposits (subcutaneous fat of legs, breast and abdominal fat) and for percentage of the main breast muscle, pectoralis major. The reverse ranking was true for leg percentage of muscle. Most of the carcass elements were still growing between the ages of 12 and 14 weeks except liver, shanks + feet, muscle and bone of legs. Differences between genetic types were found for relative growth of live weight at slaughter and carcass weight, wing weight, the weight of breast muscle (pectoralis major) and skin + subcutaneous fat of breast. Offspring from Tsaiya exhibited the highest values. Live weight percentage of various fat deposits increased between 12 and 14 weeks, along with breast muscles percentage while leg muscle percentage decreased. On average over the 4 genotypes, force-feeding increased significantly liver weight (from 55 to $583 \mathrm{~g}$ ), abdominal fat weight (from 43 to $150 \mathrm{~g}$ ), weight of skin + subcutaneous fat of legs (from 73 to $177 \mathrm{~g}$ ) and of "magrets" (from 126 to $294 \mathrm{~g}$ ). Magrets are pectoralis major with skin and subcutaneous fat of force-fed ducks. Force feeding also increased the weight of leg muscles (from 149 to $171 \mathrm{~g}$ ), but not the weight of breast muscles.

mule duck / growth / anatomical composition / cramming / crossbreeding

\section{INTRODUCTION}

L'objectif du gavage du carnard mulard, hybride inter-générique d'un canard mâle de Barbarie (Caïrina moschata) et d'une cane commune (Anas platyrhyncos), le plus souvent de type Pékin, est de produire du foie gras et du magret, muscle pectoralis major avec peau et gras sous-cutané. La station d'amélioration génétique des animaux (SAGA, INRA Toulouse) étudie le mode d'utilisation en croisement avec une cane de type Pékin d'une souche prolifique exotique, la Tsaiya brune, pour augmenter la prolificité de la mère du mulard. Cette cane est de petite taille adulte. Une expérience de croisement factoriel de cette souche avec la souche Aliénor d'origine de race Pékin produit les mères des 4 génotypes (Tsaiya, Tsaiya $x$ Pékin, Pékin $x$ Tsaiya et Pékin) utilisées ici. Nous contrôlons la croissance et la composition anatomique, avec ou sans gavage, des 4 types génétiques de mulards obtenus de leur croisement avec des mâles de Barbarie d'une souche commerciale. Razafindravao (1990) avait étudié la croissance de la naissance à la mise en gavage, la composition anato- mique aux âges de 12 et de 14 sem et la qualité technologique des foies de canetons mulards nés des mêmes souches de mères que dans notre étude, mais de pères d'une souche différente. Rouvier et al (1993 et 1994) étudient sur un échantillon plus large, mais renfermant l'échantillon traité ici, la croissance depuis la naissance et la qualité technologique des foies gras. Outre la nouveauté des types génétiques étudiés, cette expérience présente l'originalité, comme chez Auvergne (1992), d'associer l'étude d'effets abordés en général séparément, celui de l'âge (Pingel et Jeroch (1969), Ricard et al (1988)), et celui du gavage (Sazy et al, 1993).

\section{MATÉRIEL ET MÉTHODES}

\section{Matériel biologique et protocole expérimental}

Nous enregistrons les performances de 216 canetons mâles, hybrides de mâles de Barbarie et des 4 génotypes de canes communes issus d'un plan factoriel Aliénor $x$ Tsaiya brune dans l'élevage expérimental de I'INRA à Toulouse. Les 
poids adultes des canes Tsaiya, Tsaiya $\times$ Pékin, Pékin $x$ Tsaiya et Pékin sont respectivement de $1,4 \mathrm{~kg}, 2,2 \mathrm{~kg}, 2,2 \mathrm{~kg}$ et $3 \mathrm{~kg}$. Cinquante canes de chaque génotype sont fécondées par insémination artificielle avec de la semence mélangée de 2 mâles ; nous utilisons au total et simultanément 8 mâles de Barbarie sur une période d'inséminations de $9 \mathrm{sem}$. Les canetons mulards naissent en 3 lots d'éclosion les 30/10/1990, $27 / 11 / 1990,18 / 12 / 1990$. Seuls les mulards mâles sont contrôlés en élevage et en gavage à la station expérimentale des palmipèdes à foie gras d'Artiguères. À chaque lot d'éclosion, 80 mulards mâles par génotype sont conservés. lls sont élevés en cases sur caillebotis. Chaque case d'une superficie de $18 \mathrm{~m}^{2}$ comprend $12 \mathrm{~m}^{2}$ en bâtiment et $6 \mathrm{~m}^{2}$ en extérieur couvert ; elle contient 40 mulards de même génotype.

Pendant la $1^{\text {re }}$ semaine, de la $2^{\mathrm{e}}$ à la $6^{\circ}$ semaine et de la $7^{\mathrm{e}}$ à la $12^{\mathrm{e}}$ semaine, les mulards reçoivent un aliment commercial complet de composition différente. Ils sont alimentés à volonté jusqu'à l'âge de $6 \mathrm{sem}$. Du $43^{\mathrm{e}}$ au $63^{\mathrm{e}} \mathrm{j}$, l'aliment est distribué en un seul repas quotidien, en quantité limitée à $90 \%$ de la consommation mesurée pendant la $6^{\mathrm{e}}$ semaine ; du $64^{\mathrm{e}}$ au $72^{\mathrm{e}} \mathrm{j}$, la quantité distribuée est de $80 \%$ de la consommation de la $6^{\mathrm{e}}$ semaine ; du $73^{\mathrm{e}}$ au $84^{\mathrm{e}}$ jour, période de prégavage, la quantité d'aliment distribuée est augmentée de 10 à $20 \mathrm{~g} / \mathrm{jour} / \mathrm{canard}$. L'âge de mise en gavage est de $84 \mathrm{j}(12 \mathrm{sem})$; la durée du gavage n'excède pas $13 \mathrm{j}$. Les mulards sont gavés en 2 repas par jour sans repasse à l'aide de maïs mi-cuit graissé. Les mulards non gavés consomment ad libitum l'aliment de prégavage entre les âges de 12 et de 14 sem.

Par type génétique et par lot d'éclosion nous contrôlons 6 canetons mulards à l'âge de 12 sem (stade 1), à lâge de 14 sem 6 non gavés (stade 2) et 6 gavés (stade 3). Les mulards d'un génotype sont choisis parmi 80 canetons et échantillonnent la distribution des poids vifs au début du prégavage ; ils sont retenus en nombre égaux dans le premier tiers, le tiers médian et le dernier tiers des distributions.

Les mulards sont abattus en début de matinée après une diète hydrique depuis la veille au soir. Après électronarcose, les canetons sont saignés, pendus à une chaîne d'abattage, plumés, éviscérés. Les foies des canards gavés sont placés en poches de plastique sur un lit de glace pilée. Les foies et les carcasses sont stockés en chambre froide à $4^{\circ} \mathrm{C}$. Le lendemain matin a lieu la découpe des canetons ; elle est réalisée dans une salle contiguë à la chambre froide, non chauffée et à l'hygrométrie ambiante.

\section{Caractères mesurés}

A chacun des 3 stades nous mesurons le poids vif à l'abattage, le poids de la carcasse après ressuyage. Sur la carcasse nous mesurons le tour de poitirine et la longueur du bréchet. La découpe de la carcasse est réalisée selon la méthode décrite pour le poulet par Ricard et Rouvier (1967). On obtient le poids des abats consommables (gésier, foie, cœur), le poids du cou, des 2 pattes, de la tête et du gras abdominal. La cuisse et le pilon droit sont pesés puis découpés; nous retenons le poids de la peau et du gras sous-cutané, le poids du muscle et celui de l'os. On pèse les 2 ailes. On prélève et l'on pèse l'ensemble "muscles grand-pectoraux + peau + gras sous-cutané», appelé "magret» chez l'animal gravé, puis séparément l'ensemble "peau + gras sous-cutané» et les muscles grand-pectoraux. Les 2 muscles petit-pectoraux sont détachés du bréchet et pesés.

Nous avons calculé les proportions de tous les caractères mesurés par rapport au poids vif ainsi que les proportions des éléments de la cuisse et des filets par rapport au poids de la cuisse et des filets respectivement.

\section{Méthodes statistiques}

Nous analysons séparément les stades 1 et 2 d'une part et le stade 3 d'autre part car les variances chez les canetons gavés sont supérieures.

Une analyse de variance croisée à effets fixés est réalisée pour les stades 1 et 2 (modèle 1) en considérant les effets du type génétique (4 modalités), du lot d'éclosion ( 3 modalités) et du stade (2 modalités) et l'interaction entre le type génétique et le stade.

Nous analysons les performances mesurées, ou transformées en valeur logarithmique, ou exprimées en proportion. L'analyse des proportions présente un inconvénient statistique : ces variables n'ont pas une distribution normale. Mais cette transformation nous semble préférable à un ajustement linéaire car, pour les types génétiques eg, les différences de poids moyen induisent des dis- 
tributions disjointes des autres caractères ; l'ajustement conduirait alors à extrapoler des variables en dehors de leurs limites biologiques de variation. L'analyse des valeurs logarithmiques permet d'exprimer la croissance relative du poids vif, des organes et des tissus. En effet, par définition de la dérivabilité de la fonction Logarithme, dLogy/dy $\rightarrow 1 / y$ lorsque $d y \rightarrow 0$; pour un $d y$ suffisamment petit $d \log y=\mathrm{dy} / \mathrm{y} ;$ or $d y / y$ est par définition la croissance relative au voisinage d'y. Les données étant acquises par abattage des animaux, un individu contrôlé à l'âge de 12 sem n'a pas d'individu avec qui s'apparier à l'âge de $14 \mathrm{sem}$. Nous calculons la différence des valeurs moyennes des données transformées aux 2 premiers stades qui, selon la formule ci-dessous, exprime la croissance relative :

$$
\begin{gathered}
M(\log y 2)-M(\log y 1)=2(M(y 2)-M(y 1)) \\
/(M(y 2)+M(y 1))
\end{gathered}
$$

où $M$ signifie valeur moyenne.

En faisant le rapport des accroissements moyens des valeurs logarithmiques d'une variable par rapport au poids de carcasse, on exprime le rapport des croissances relatives, ce qui donne une idée de l'allométrie de croissance. En effet ce rapport s'exprime comme :

$$
M(\log y 2)-M(\log y) / M(\log x 2)-M(\log x 1)
$$

et représente la pente de la droite joignant les moyennes aux âges de 12 et de 14 sem des données transformées. Cette approximation de l'allométrie de croissance est d'autant meilleure que la régression intra-stade est proche de la régression entre-stades et que les caractères sont moins variables. Dire que la régression intra-stade est proche de la regression entre-stades, c'est dire qu'il n'y a pas de modification de la vitesse de croissance relative entre les 2 stades.

Nous analysons les caractères des animaux gavés à l'aide d'un modèle d'analyse de variance croisé à effets fixés incluant l'effet du type génétique, du lot d'éclosion et de leur interaction (modèle 2).

Nous utilisons une statistique $t$ de Student pour comparer les moyennes entre stades intragénotype (modèle 1) et pour les canetons gavés (modèles 1 et 2) ; nous utilisons une statistique $t$ généralisée de Bonferroni pour comparer les moyennes entre génotypes intra-stade. Les calculs sont réalisés sur l'ordinateur IBM 30.90 du centre de traitement de l'information de Jouy-en-
Josas à l'aide de logiciels de la programmathèque SAS.

\section{RÉSULTATS}

Nous allons successivement étudier la composition corporelle à 12 et à 14 sem, les variations de 12 à 14 sem puis l'effet du gavage. Les tableaux I et II donnent les moyennes des moindres carrés des poids des éléments de la carcasse par génotype et par stade (modèles 1 et 2). Ils donnent également la signification statistique des effets stade et d'interaction génotype $x$ stade dans le modèle 1 , sur les valeurs initiales et sur les valeurs logarithmiques. Le tableau III donne les moyennes des moindres carrés des proportions des éléments de la carcasse et la signification des effets génotype et stade (12 et 14 sem). Le tableau IV donne la croissance relative des différentes parties de la carcasse ainsi que la valeur de leur rapport.

\section{Composition corporelle à 12 et 14 sem}

Aux âges de 12 et de 14 sem, les différences entre les types génétiques sont significatives pour tous les caractères. Les mulards de Tsaiya sont les plus légers pour tous les caractères; les mulards de canes métisses sont intermédiaires ; ceux de canes Pékin les plus lourds.

Le type génétique influence les proportions par rapport au poids vif autres que celles de la cuisse droite, des ailes et des muscles petits pectoraux.

Proportionnellement au poids vif, les mulards de Tsaiya ont plus de muscle et moins de gras sous-cutané de la cuisse que les mulards de Pékin ; les mulards de canes métisses sont intermédiaires. Les mulards de Tsaiya ont également une proportion moins importante de peau + gras des filets 
et de gras abdominal que les mulards de Pékin ; les mulards de canes métisses sont intermédiaires. La proportion des muscles pectoraux dans la carcasse est plus faible chez les mulards de Tsaiya que chez les Pékin. Pour la proportion de muscle dans le filet ou dans la cuisse, les types génétiques se classent ainsi : Tsaiya $>$ Pékin $x$ Tsaiya $=$ Tsaiya $\mathrm{x}$ pékin $>$ Pékin .

\section{Croissance entre les âges de 12 et $14 \mathrm{sem}$}

Les valeurs des caractères autres que le foie, les pattes, le muscle et l'os de la cuisse s'accroissent significativement entre les âges de 12 et 14 sem.

L'analyse de variance des Log confirme ce résultat. Elle fait en outre apparaître des interactions génotype $x$ stade significatives pour le poids à l'abattage, les poids de carcasse ressuyée et éviscérée, le tour de poitrine et les poids de l'ensemble «peau + gras sous-cutané" de la cuisse et des filets, les poids des 2 ailes, des filets et des muscles grand-pectoraux des filets. Cela traduit une croissance relative de ces caractères différente d'un génotype à un autre ; celle-ci est plus forte pour les mulards de Tsaiya (tableau IV). En moyenne pour les 4 types génétiques, la croissance relative des organes et tissus varie de 7,5 (ailes) à $84,6 \%$ (gras abdominal). Entre 12 et $14 \mathrm{sem}$, l'ordre de développement des tissus des organes est le même pour les 4 types génétiques ; l'os et le muscle de la cuisse ont cessé de croître. Les rapports de la croissance relative des organes à la croissance relative de la carcasse sont de 0,7 pour les ailes, 0,8 pour l'ensemble de la cuisse, 1,2 pour les muscles grand-pectoraux, et 1,3 pour les petits pectoraux, 2,4 et 2,5 pour l'ensemble peau + gras sous-cutané de la cuisse et des filets et 7,8 pour le gras abdominal. Ainsi notre approximation de l'allométrie de croissance entre 12 et 14 sem correspond à une allo- métrie minorante pour le poids des ailes et de la cuisse, majorante pour les muscles petit et grand-pectoraux et fortement majorante pour le tissu gras.

L'analyse des proportions indique un accroissement significatif entre 12 et $14 \mathrm{sem}$ pour le pourcentage de carcasse ressuyée, de carcasse éviscérée, le pourcentage de gras abdominal et de l'ensemble «peau + gras sous-cutané" des filets et de la cuisse. Les proportions d'os et de muscle de la cuisse diminuent alors que celle du muscle du filet augmente.

\section{Effets du gavage}

Intra-type génétique, le poids du gésier, celui de l'os de la cuisse, celui des muscles petits et grands pectoraux sont égaux que les canetons soient gavés ou non. Les poids du cou, de la tête et la longueur du bréchet sont plus élevés chez les mulards de Pékin gavés. Pour tous les autres caractères, le gavage augmente les poids ou les dimensions dans chaque génotype, à la seule exception du poids des 2 ailes des mulards de métisses Tsaiya $\times$ Pékin. En valeurs absolues, le poids du foie subit la plus forte augmentation passant de $55 \mathrm{~g}$ à $583 \mathrm{~g}$ en moyenne sur l'ensemble des 4 types génétiques; ensuite c'est le gras abdominal (de 43 à $150 \mathrm{~g}$ ) puis l'ensemble "peau + gras" de la cuisse (de 73 à $177 \mathrm{~g}$ ) et celui des 2 magrets (de 126 à $294 \mathrm{~g}$ ). On note également un accroissement du poids des pattes (de 85 à $106 \mathrm{~g}$ ). Le poids de muscle de la cuisse s'accroît de 149 à $171 \mathrm{~g}$. Il en résulte une augmentation de $51 \%$ du poids de la cuisse (alors que celle du poids des ailes n'est que de $8 \%$ ), et de $41 \%$ du poids de carcasse éviscérée.

Chez les animaux gavés les différences entre génotypes sont significatives pour tous les caractères. Le classement des génotypes fait apparaître selon le caractère soit 3 groupes, soit 2 groupes de génotypes. 


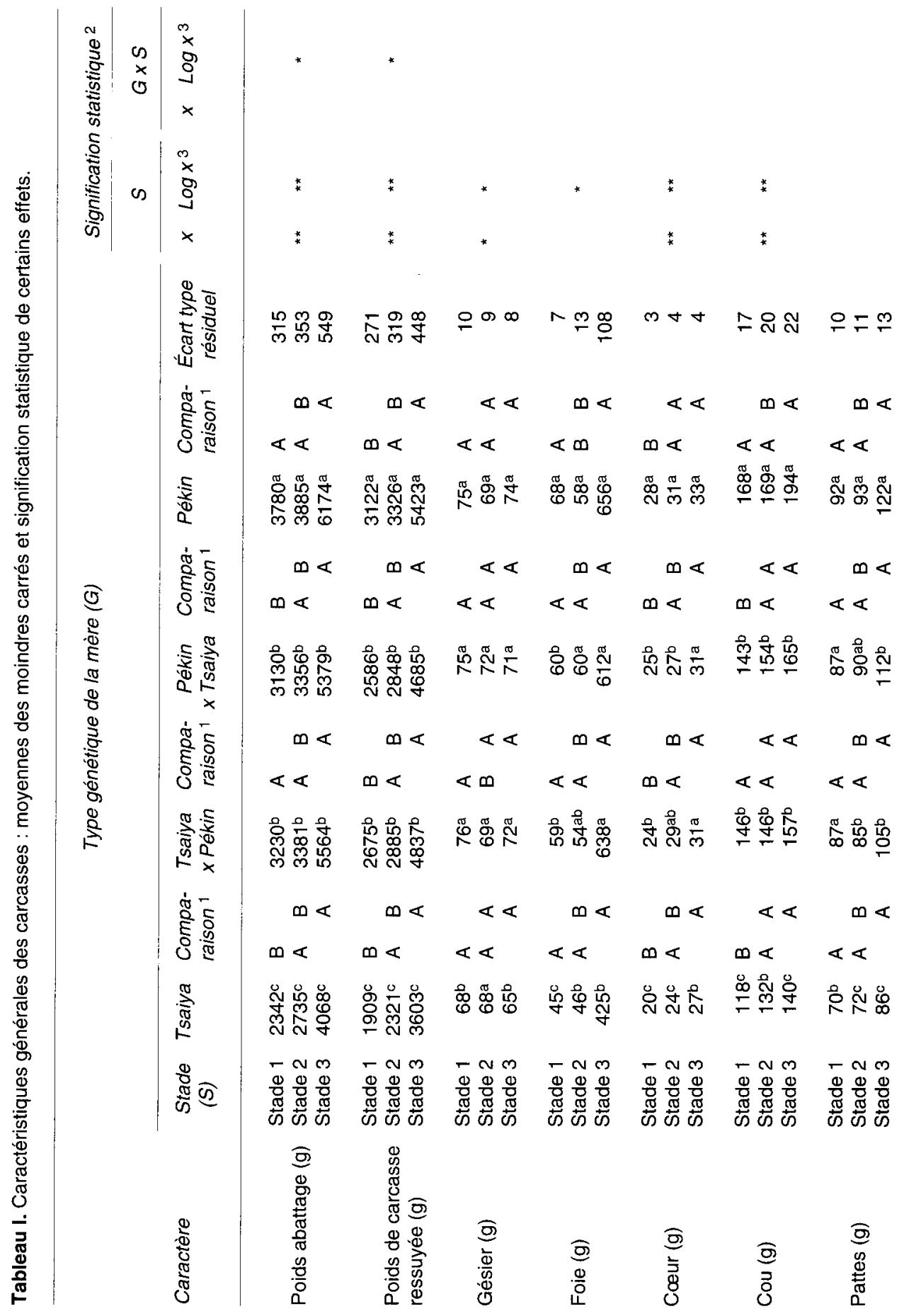




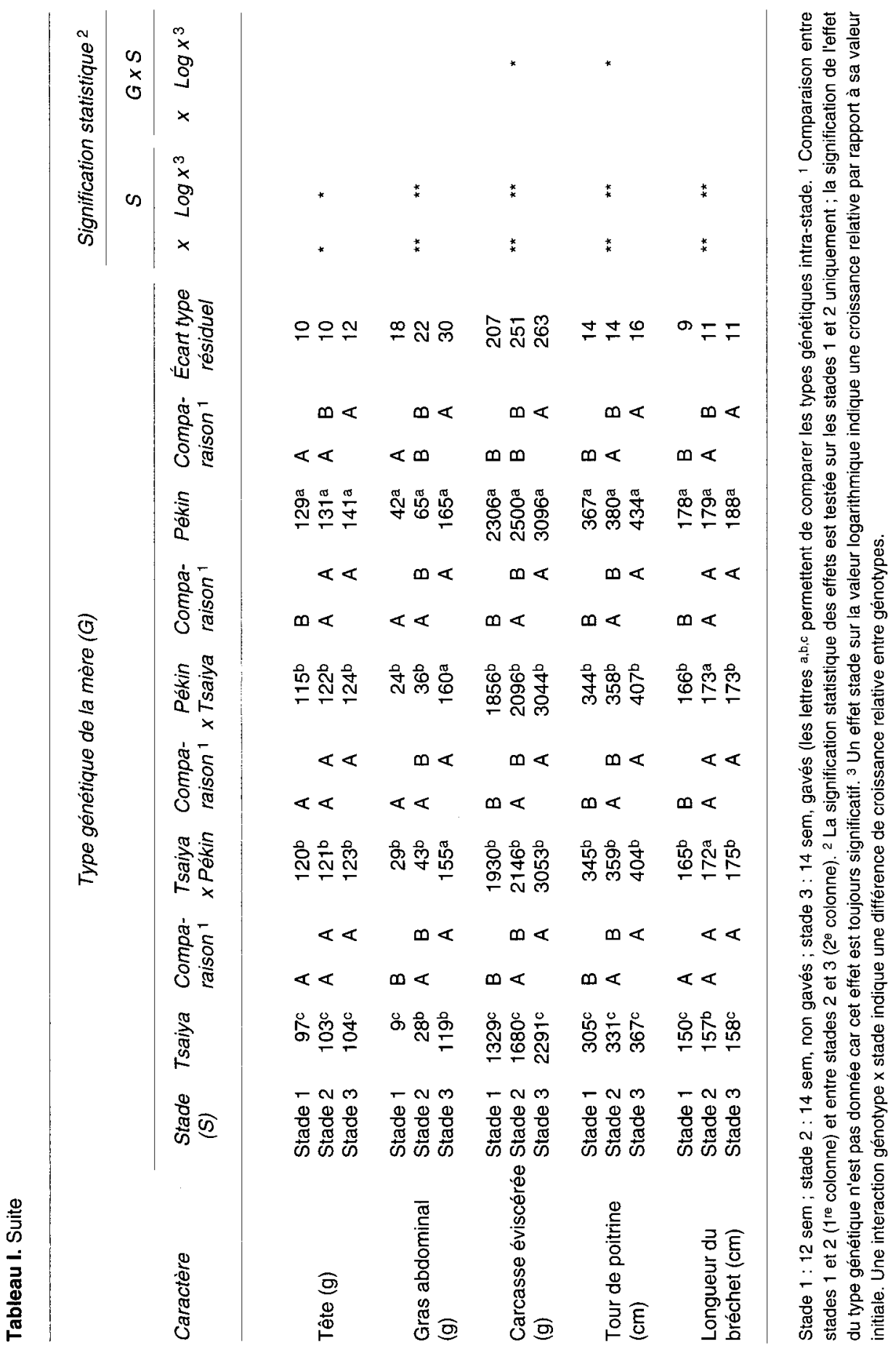




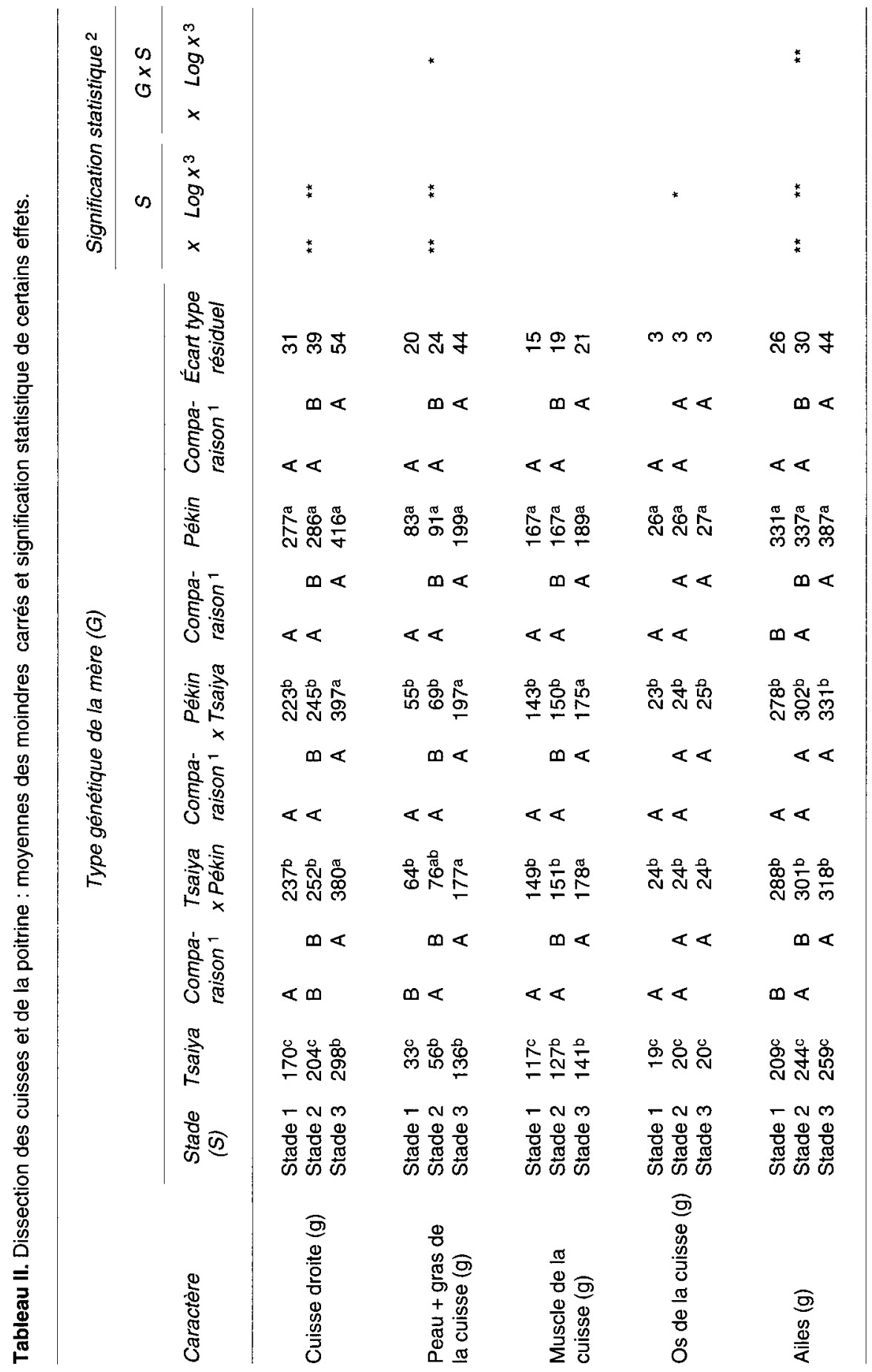



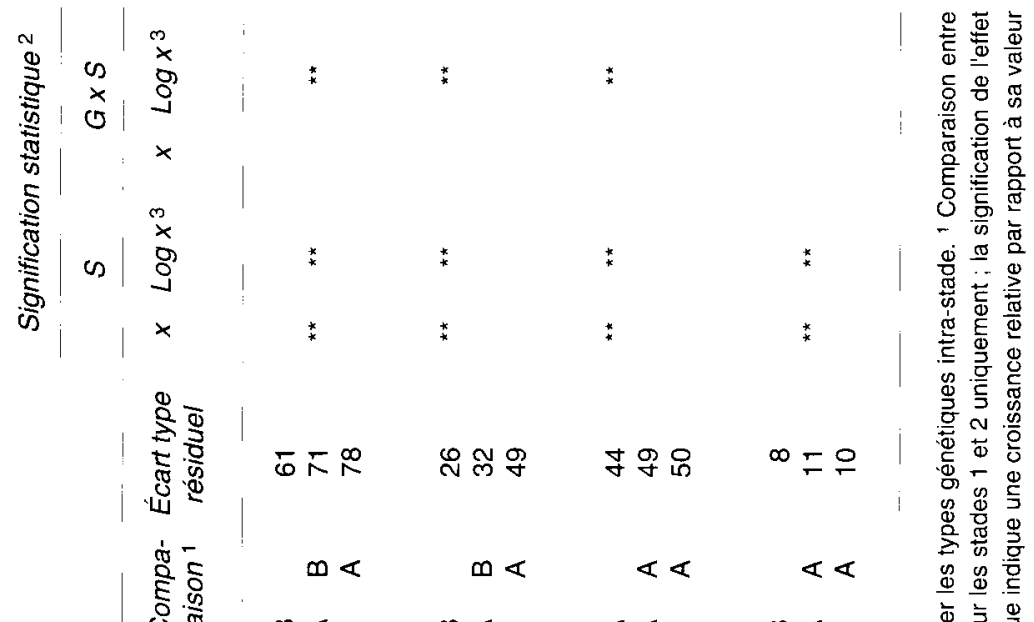

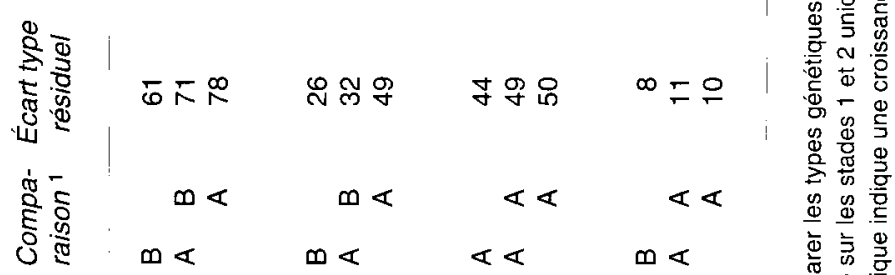

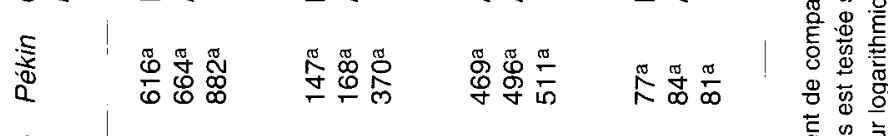

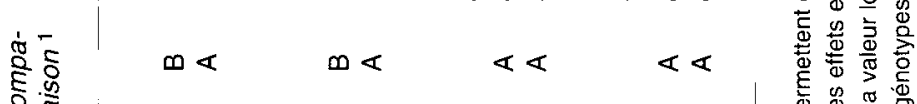

(1) $\mid$ S



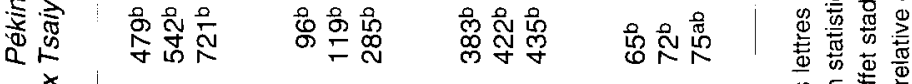

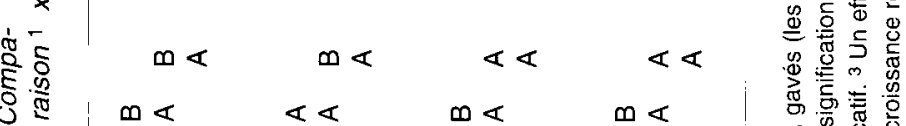

$\infty \ll \quad \varangle \ll \quad \omega \ll \quad \infty \ll \quad$ 焉

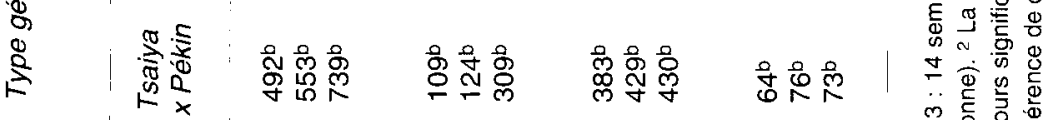

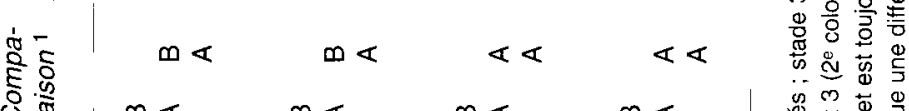

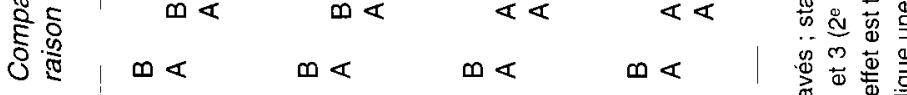

I

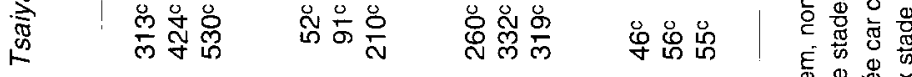

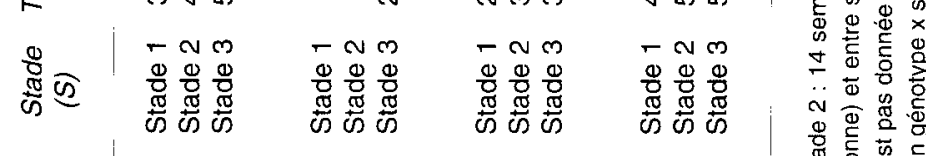

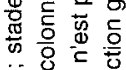

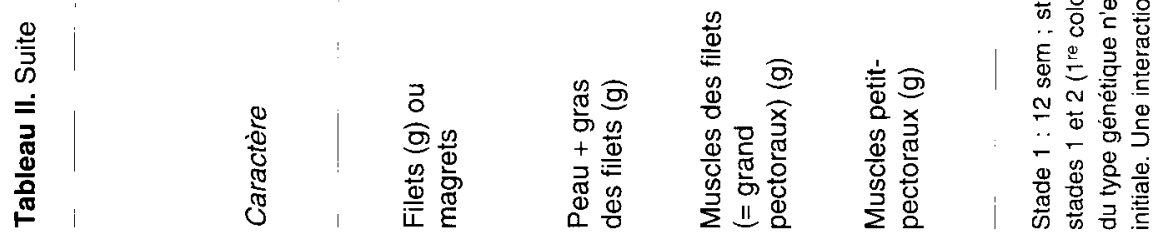




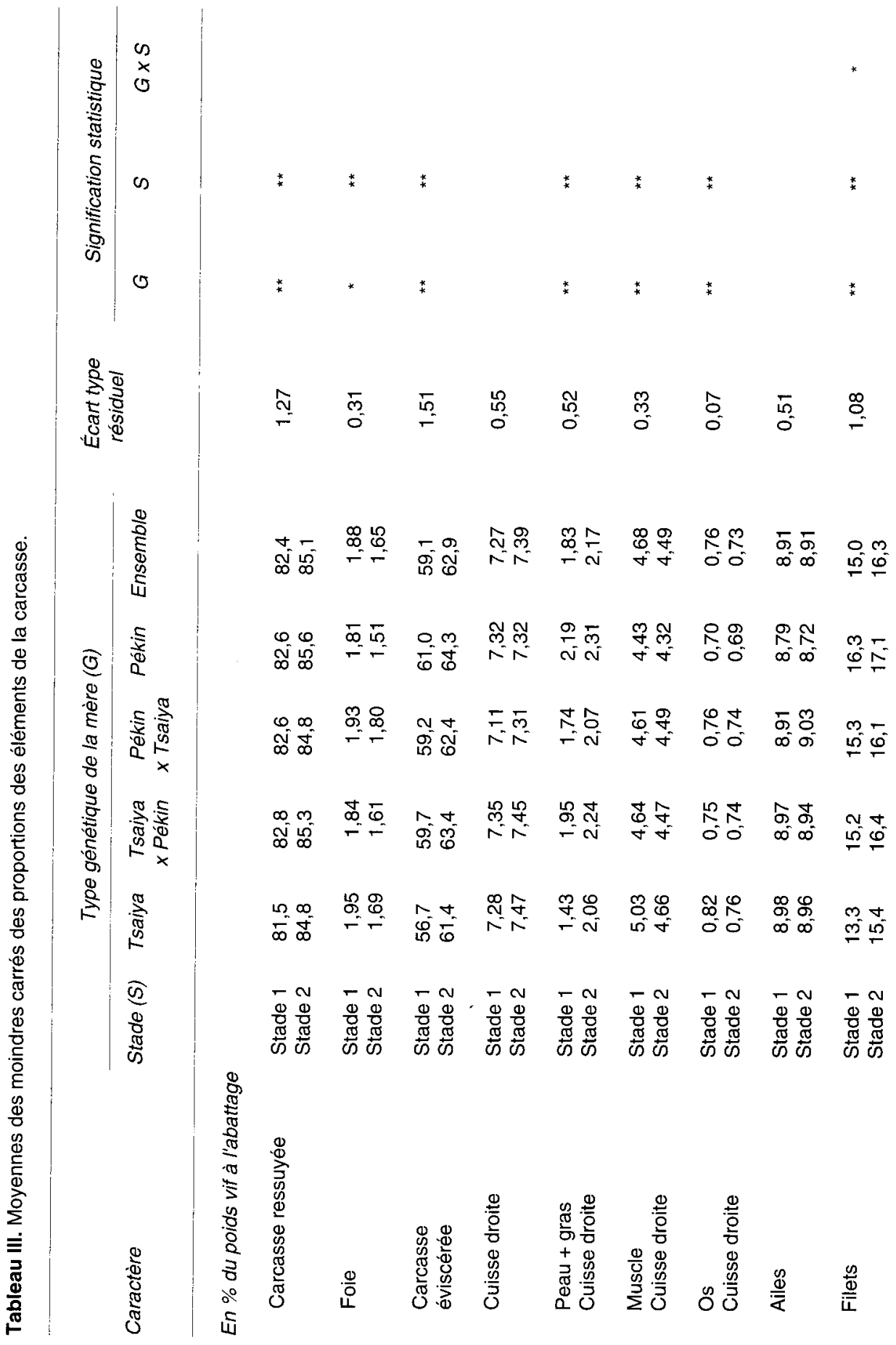




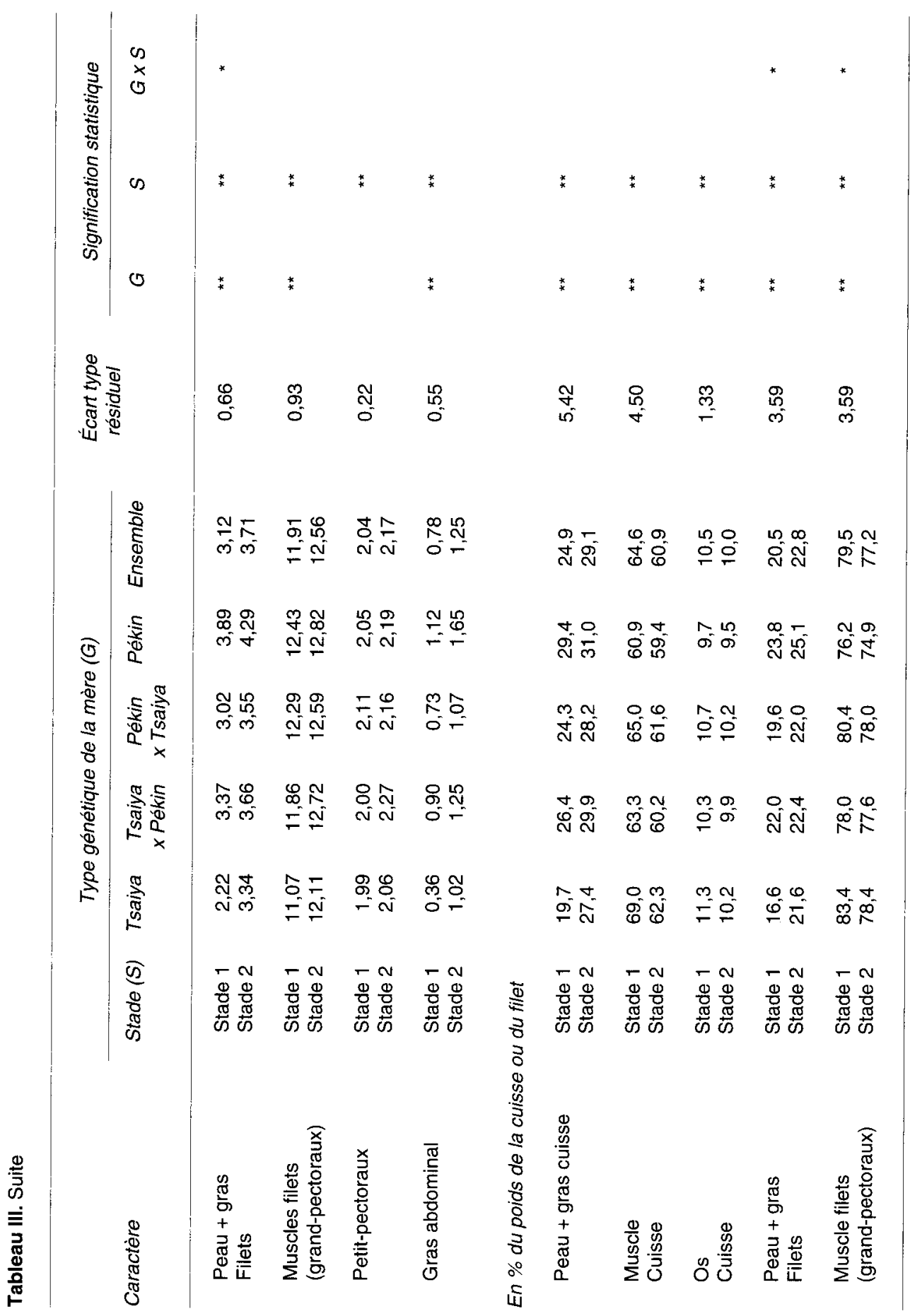


Tableau IV. Croissance relative ${ }^{a}$ de différentes parties de la carcasse (1re ligne) et coefficients d'allométrie $^{\mathrm{b}}$ de croissance ( $2^{\mathrm{e}}$ ligne) entre les âges de 12 et $14 \mathrm{sem}$.

\begin{tabular}{|c|c|c|c|c|c|}
\hline & & Type génétiqu & e de la mère & & Moyenne \\
\hline & Tsaiya & Tsaiya x Pékin & Pékin x Tsaiya & Pékin & \\
\hline Poids à l'abattage & 15,8 & 4,6 & 7,3 & 2,6 & 7,6 \\
\hline & - & - & - & - & - \\
\hline Carcasse ressuyée & 19,8 & 7,4 & 10,0 & 6,1 & 10,9 \\
\hline & - & - & - & - & - \\
\hline Carcasse éviscérée & 23,8 & 10,6 & 12,5 & 7,8 & 13,7 \\
\hline & 1,2 & 1,4 & 1,3 & 1,3 & 1,3 \\
\hline Gras abdominal & 150,4 & 69,9 & 59,0 & 58,9 & 84,6 \\
\hline & 7,6 & 9,4 & 5,9 & 9,7 & 7,8 \\
\hline Peau + gras sous-cutané & 51,8 & 20,0 & 26,3 & 8,2 & 26,0 \\
\hline de la cuisse & 2,6 & 2,7 & 2,6 & 1,3 & 2,4 \\
\hline Peau + gras sous-cutané & 58,0 & 15,0 & 24,8 & 12,1 & 27,5 \\
\hline des filets & 2,9 & 2,0 & 2,5 & 2,0 & 2,5 \\
\hline Muscles grand-pectoraux & 24,6 & 11,6 & 9,5 & 5,5 & 12,8 \\
\hline & 1,2 & 1,6 & 1,0 & 0,9 & 1,2 \\
\hline Muscles petit-pectoraux & 19,5 & 16,6 & 9,4 & 9,1 & 13,7 \\
\hline & 1,0 & 2,2 & 0,9 & 1,5 & 1,3 \\
\hline Ailes & 15,9 & 5,4 & 8,4 & 1,0 & 7,5 \\
\hline & 0,8 & 0,7 & 0,8 & 0,2 & 0,7 \\
\hline Cuisse droite & 18,3 & 6,1 & 9,7 & 2,3 & 9,1 \\
\hline & 0,9 & 0,8 & 1,0 & 0,4 & 0,8 \\
\hline
\end{tabular}

a Accroissement relatif du poids $(y)$ entre les stades 1 et 2 . Calculé comme $\Delta \log y=[M(\log y 2)-M(\log y 1)] x$ 100 , où $M$ signifie valeur moyenne ; ${ }^{b} \Delta \log y / \Delta \log x$ où $y$ et $x$ désignent respectivement le poids d'un organe et le poids de carcasse ressuyée.

Les 3 groupes sont: les génotypes de mères Pékin, qui donnent les valeurs les plus élevées des caractères, ceux des mères métisses, qui ont des valeurs intermédiaires, ceux de mères Tsaiya, qui donnent les valeurs les plus faibles. Les 2 groupes sont d'une part les génotypes de mères Pékin et des 2 métisses réciproques, d'autre part le génotype de mère Tsaiya dont les mulards ont les valeurs les plus faibles. II s'agit des caractères de poids du gésier, du foie gras, du cœur, du gras abdominal, de la cuisse, des peaux + gras sous cutané et muscle de la cuisse. 


\section{DISCUSSION}

Au niveau de l'analyse de variance, nous constatons que tout test significatif en données brutes l'est en données transformées en logarithmes ; que des tests, en particulier d'interactions, non significatifs en données brutes le deviennent en données transformées ; or, selon Kendal et Stuart (1966), la première vertu de la transformation logarithmique est d'homogénéiser les variances donc d'augmenter la précision des tests. Théoriquement, toutes hypothèses de l'analyse de variance vérifiées, la transformation ne devrait pas induire des changements de signification d'un modèle à l'autre. Le dispositif expérimental est équilibré, l'indépendance en échantillonnage au moins des effets considérés n'est pas douteuse. Une surestimation de la variance résiduelle des données brutes est alors l'hypothèse la plus vraisemblable. Cette situation se rencontre lorsque les variances par cellule ne sont pas égales. Entre les âges de 12 et de 14 sem, les variances des caractères augmentent.

Nous approximons le coefficient d'allométrie de croissance entre les âges de 12 et de 14 sem par le rapport des différences de valeurs moyennes transformées d'un organe ou d'un tissu aux différences de valeurs moyennes transformées du poids de carcasse. Lerner (1938a, b) utilise ce même rapport pour des valeurs individuelles ; il le dénomme "rapport de croissance relative»; il montre que ce rapport estime le coefficient d'allométrie de la fonction de croissance de Huxley et Teissier (1936).

Entre les âges de 12 et de 14 sem, les mulards de canes Pékin, les plus lourdes, ont la plus faible croissance et ceux de Tsaiya, les plus légères, la plus forte. En revanche jusqu'à l'âge de 12 sem les différences de croissance correspondent aux différences de poids adulte des mères. II faut cependant analyser les croissances relatives et les variations de proportion des tissus entre types génétiques.

Le classement des organes et des tissus, de la croissance la plus précoce à la croissance la moins précoce, est : l'os, le muscle de la cuisse, les ailes, la cuisse, les muscles pectoraux, l'ensemble peau et gras sous-cutané de la cuisse et des filets, le gras abdominal. Sur le plan zootechnique un abattage à un poids plus élevé permettrait donc d'accroître la quantité de muscles pectoraux, partie noble de la carcasse, mais augmenterait également la quantité de tissu gras.

Comme le font observer Auvergne (1992) et Ricard et al (1988), le canard se distingue du poulet ou de la pintade par un développement non synchrone des cuisses et des filets. Ricard et al (1988) situent à l'âge de 8 sem la fin de la croissance musculaire de la cuisse; nous le situons à 12 sem, âge le plus précoce contrôlé ici. Le muscle du filet poursuit sa croissance au-delà de cet âge avec des vitesse relatives variables d'un génotype à un autre, $5 \%$ chez les mulards de Pékin, $25 \%$ chez les mulards de Tsaiya.

La croissance relative du poids vif entre 12 et 14 sem constitue un indicateur du stade de développement corporel. À un âge donné, une croissance relative plus élevée signale un développement plus tardif. Du plus précoce au moins précoce, les 4 génotypes se classent dans l'ordre suivant: Pékin, Pékin x Tsaiya, Tsaiya x Pékin et Tsaiya. Les mulards issus des mères ayant le plus grand format adulte sont ainsi les plus précoces pour la croissance corporelle globale entre les âges de 12 et de 14 sem. Les mulards de mères Pékin ont également un développement plus précoce des tissus adipeux, des muscles pectoraux, des ailes et de la cuisse. Ces mulards ont donc un pourcentage plus important de gras et de muscles pectoraux. En revanche, les mulards de Tsaiya, du fait de leur moindre précocité, ont un pourcentage de muscle de la cuisse supérieur. 
Outre les effets escomptés du gavage, augmentation du poids du foie et des dépôts gras sous-cutanés et abdominaux, on observe une augmentation du poids des muscles de la cuisse. Ce que nous appelons muscles de la cuisse est un ensemble de muscles entre lesquels du tissu adipeux peut se loger. La croissance musculaire de la cuisse étant terminée au moment de la mise en gavage, l'augmentation du poids des muscles de la cuisse refléterait alors une accumulation de gras intra ou péri-musculaire. Auvergne (1992) et Sazy et al (1993) n'observent pas ce phénomène en gavage ; Auvergne (1992) l'interprète par le peu de sensibilité aux conditions nutritionnelles de muscles dont la croissance est terminée. Nous observons un gain de poids identique des muscles pectoraux entre 12 et 14 sem chez les animaux maigres et chez les animaux gavés. Ce résultat diffère également de celui d'Auvergne (1992) qui observait un ralentissement de la croissance des pectoraux sous l'effet du gavage par rapport aux animaux maigres contemporains et l'interprétait par la compétition entre le muscle pectoral et le foie pour les rapports azotés nutritionnels. Dans notre cas, ce phénomène n'apparaît pas, à moins d'admettre également des dépôts adipeux intra-musculaires qui compenseraient, le cas échéant, le déficit de croissance protéique des muscles pectoraux.

On peut aussi suspecter que le rationnement mis en place a été un peu trop sévère. En effet, nous avons contrôlé la consommation entre 5 et 6 sem à une période où les animaux venaient tout juste d'avoir accès au parcours extérieur. Dans un premier temps, ils se sont montrés réticents à utiliser cette possibilité et sont restés dans le local intérieur chauffé. Les consommations mesurées alors ont été relativement modestes. Lors de la période de rationnement, les canards ont largement utilisé le parcours extérieur ; les températures étaient basses ; les canards ont donc utilisé une partie de leur ration pour assurer leur thermorégulation. Ceci s'est traduit par un retard de croissance probable dans tous les génotypes (à titre d'exemple, un mulard de Pékin pèse de 4000 à $4100 \mathrm{~g}$ à l'âge de $12 \mathrm{sem}$ au lieu des $3780 \mathrm{~g}$ que nous avons enregistrés ici). La pénalisation n'a peut-être pas été équivalente pour tous les génotypes et a pu avoir une influence sur leurs précocités respectives. En tout état de cause, ce phénomène explique probablement les différences de résultats par rapport aux auteurs cités précédemment, le gain de poids observé entre 12 et 14 sem étant alors imputable à une croissance compensatrice.

\section{CONCLUSION}

Aux âges de 12 et de 14 sem, le poids corporel des 4 génotypes de mulards ainsi que le poids des éléments de leur carcasse reflètent les différences de format des mères. Les mulards des canes Pékin sont les plus lourds, les mulards des Tsaiya les plus légers, les mulards des canes métisses, intermédiaires. On observe cependant des différences de composition anatomique et tissulaire des carcasses. Celles-ci semblent en grande partie résulter des différences de maturité corporelle entre les types génétiques. Les mulards de Pékin, les plus précoces ont une proportion plus importante que les Tsaiya d'organes et de tissus à développement tardif comme les muscles pectoraux, le gras sous-cutané et le gras abdominal. Les mulards de Tsaiya, les plus tardifs, ont une proportion plus importante de muscles de la cuisse dont la croissance est terminée à l'âge de 12 sem. Dans les 4 génotypes, le gavage augmente non seulement le poids du foie et de divers tissus gras mais aussi celui des muscles de la cuisse par accumulation de gras intra- ou péri-musculaire. Il ne pénalise pas la croissance des muscles pectoraux, contrairement à des résultats antérieurs (Auvergne, 
1992), bien qu'il ait pu en diminuer le rapport protéines/lipides.

\section{REMERCIEMENTS}

Les auteurs remercient le Taiwan Livestock Research Institute (République de Chine) qui a fourni les canards de la souche Tsaiya brune dans le cadre d'une coopération scientifique. Is remercient également la SICA-SEPALM, centre de sélection des palmipèdes à foies gras ( $F 40250$, Souprosse), qui a fourni les reproducteurs de la souche Aliénor pour cette expérience. Ils remercient pour leur participation à la réalisation expérimeniale les techniciens animaliers de la station expérimentale des palmipèdes à fole gras à Artiguères ( $M$ Ducourneau, D Gourichón, JC Momas), les agents et les techniciens animaliers de la station expérimentale lapins et palmipèdes (F Tudela, C Bellio, F Ruiz).

\section{RÉFÉRENCES}

Auvergne A (1992) Facteurs de variation de la composition corporelle et tissulaire des canards avant et après gavage. Thèse doct, Institut national polytechnique de Toulouse, $252 \mathrm{p}$

Huxley JS, Teissier G (1936) Terminology of relative growth. Nature 137, 780-785

Kendall MA, Stuart A (1966) Advanced theory of statistics. Griffin, London, vol 3, ch 37, 88-96

Lerner IM (1938a) Growth ratio of the fowl's tarsometatarsus. I. Variation with respect to body weight. Growth 2, 135-140
Lerner IM (1938b) Growth ratio of the fowl's tarsometatarsus. II. Variation with respect to time. Growth 2, 141-144

Pingel $H$, Jeroch $H$ (1969) Untersuchungen über die Mast und Schlachtleistung sowie die Fleich qualität von Tieren der Kreuzung Moschuserpel Pekingente. Arch Geflügelz Kleint 18, 285-292

Razafindravao J (1990) Étude comparative des performances zootechniques de 7 types génétiques de canes communes (Anas platyrhyncos) en production de mulards. Mémoire de DEA, École nationale supérieure agronomique de Toulouse, $49 \mathrm{p}$

Ricard FH, Rouvier R (1967) Étude de la composition anatomique du poulet. I. Variabilité de la répartition des différentes parties corporelles chez les coquelets Bresse-Pile. Ann Zootech 16, 23-29

Ricard FH, Carville H de, Marche G (1988) Étude comparative de la composition anatomique des canards de Barbarie, Pékin et mulard. In : La génétique du canard de Barbarie et du mulard ( $R$ Rouvier, ed), Toulouse - Auzeville, 15-16 octobre 1985, Les colloques de I'INRA 42, 75-99

Rouvier R, Guy F, Rousselot-Pailley D, Poujardieu B (1993) Effets génétiques du croisement de souches Tsaiya brune et Pékin sur la croissance et le foie gras de leurs mulards mâles. Proc 1res Journées de la recherche sur les palmipèdes à foie gras, Bordeaux, 28 avril 1993, ITAVI (ed), 3-12

Rouvier R, Guy G, Rousselot-Pailley D, Poujardieu B (1994) Genetic parameters from factorial crossbreeding in 2 common duck strains (Anas platyrhynchos) Brown Tsaiya and Pekin, for growth and fatty liver traits in their male mule duck progeny. $\mathrm{Br}$ Poul Sci 35, 509-517

Sazy E, Heraut F, Robin N (1993) Évolution de la composition corporelle des canards mulards pendant le gavage. Proc yres Journées de la recherche sur des palmipèdes à foie gras, Bordeaux, 29 avril 1993, ITAVI (ed), 45-61 